\title{
40-GHz MMIC SPDT and Multiple-Port Bandpass Filter-Integrated Switches
}

\author{
Shih-Fong Chao, Student Member, IEEE, Che-Chung Kuo, Zou-Min Tsai, Student Member, IEEE,
} Kun-You Lin, Member, IEEE, and Huei Wang, Fellow, IEEE

\begin{abstract}
GHz monolithic microwave integrated circuit (MMIC) single-pole-double-throw and multiple-port bandpass filter-integrated switches based on electronically switchable resonators are proposed. The proposed multifunction chip integrates a multiple-port switch with bandpass filter functions in a single chip. The switchable resonators are formed by quarter-wavelength stepped-impedance resonators with passive HEMT loading at one end. By properly allocating the resonant frequencies of the resonators in their on and off modes, a filter-integrated switch can perform a bandpass response with spurious suppression in the on state and achieve wideband isolation in the off state. The technique of using shared resonators is also introduced in the circuit design to reduce the overall circuit size. The results show the proposed circuits successfully integrate a MMIC switch with bandpass filter functions into a single circuit component.
\end{abstract}

Index Terms-Bandpass filter, monolithic microwave integrated circuit (MMIC), multiple port, single-pole double-throw (SPDT), stepped-impedance resonator (SIR), switch.

\section{INTRODUCTION}

$\mathbf{T}$ HE MICROWAVE multifunction integration has always been a popular subject [1]-[4]. As the commercial and military systems become more complex, more functions are desired in a smaller chip area. A microwave switch is an essential building block at the RF front end for time-division duplexing (TDD) communication systems. Recently, several passive switches using field-effect transistors (FETs) or $\mathrm{p}-\mathrm{i}-\mathrm{n}$ diodes have been reported [5]-[12]. However, most of these switches target for wideband design, implying that their operating bandwidths are usually over $50 \%$ and cannot provide sharp band rejections. Therefore, a preselected bandpass filter will be needed to cascade with such a switch to provide band selectivity and reject out-of-band signals for system applications.

The concept of filter-integrated switch circuits were reported in [13]-[16]. In [13], a ring resonator loaded with a p-i-n diode was developed as a switchable filter. However, it occupied a large layout size, and a high-order implementation is difficult. In

Manuscript received April 23, 2007; revised September 6, 2007. This work was supported in part by the National Science Council under Grant NSC 95-2218-E-002-057, Grant 95R0062-AE00-01, and Grant NSC 94-2213-E-002-008-PAE

The authors are with the Graduate Institute of Communication Engineering and the Department of Electrical Engineering, National Taiwan University, Taipei 106, Taiwan, R.O.C. (e-mail: kunyou@ntu.edu.tw; hueiwang@ntu.edu.tw).

Color versions of one or more of the figures in this paper are available online at http://ieeexplore.ieee.org.

Digital Object Identifier 10.1109/TMTT.2007.909142
[14], switchable filters were reported based on stub filter structures, the switching device is used to change the boundary condition of each periodically loaded stub in the on and off states. The filter-integrated switch concept is also applied to monolithic microwave integrated circuit (MMIC) circuit designs in [15] and [16]. In [15], the image-filter synthesis method is used to design a wideband switch. In [16], a filter-integrated switch based on stub filter structures are proposed for wideband applications. However, the above-mentioned designs only focus on the passband, which means that only the on-state filter response and off-state isolation in the vicinity of the center frequency were considered. Therefore, they would suffer from unwanted spurious responses and narrow band isolation in the on and off states, respectively. In [17], filter-integrated switches based on diode-loaded stepped-impedance resonators (SIRs) were proposed to achieve a bandpass response with a wide stopband rejection in the on state and a wideband isolation in the off state.

To integrate switches and bandpass filters at the same chip level can not only save the additional losses caused by the off-chip interface, but also make high-level system-on-chip (SOC) integrations possible. In this paper, the concept of the filter-integrated switch in [17] is extended to design a compact MMIC single-pole-double-throw (SPDT) [18] and a compact multiple-port filter-integrated switches. The hybrid filter-integrated switches in [17] are designed based on half-wavelength loaded SIRs. In order to reduce the circuit size, switchable quarter-wavelength SIRs are chosen to implement a 40-GHz MMIC SPDT and a multiple-port filter-integrated switches. The switchable quarter-wavelength resonator is formed by loading a passive HEMT device at the open end of a quarter-wavelength SIR. By applying different control voltage, the resonant conditions of the loaded resonator can be changed. The advantages of a loaded quarter-wavelength SIR over a half-wavelength SIR is its compact size. Moreover, the span between adjacent resonant frequencies is twice wider than that of a half-wavelength resonator. Therefore, it is more flexible to allocate the resonant frequencies of each resonator in both on and off states. To further reduce the overall circuit size, the shared resonator technique is also used to save the number of resonators and replace the conventional matching $\mathrm{T}$-junction in a millimeter-wave switch design.

\section{MMIC PROCESS}

The process used in this design is WIN Semiconductors' $0.15-\mu \mathrm{m}$ high-linearity AlGaAs/InGaAs/GaAs pseudomorphic HEMT (pHEMT) MMIC process. The thickness of the GaAs substrate is 4 mil with a dielectric constant of 12.9. In this passive HEMT switch design, the total gatewidth of the device 


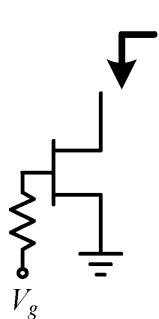

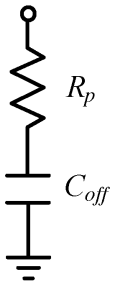

(a)

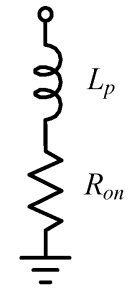

(b)
Fig. 1. Equivalent-circuit models for a passive HEMT. (a) $V_{g}=-3 \mathrm{~V}$. (b) $V_{g}=0 \mathrm{~V}$.

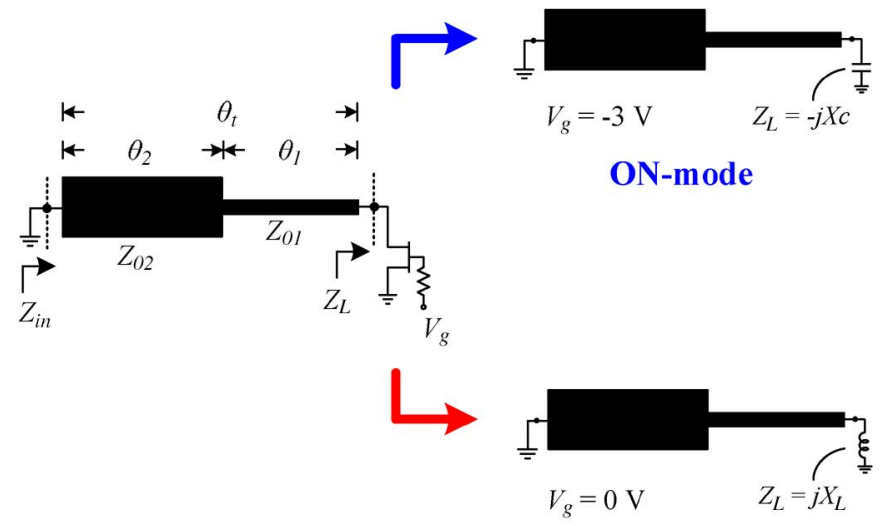

OFF-mode

Fig. 2. Circuit configuration of the proposed HEMT loaded SIR and its equivalent on- and off-mode resonators.

is $60 \mu \mathrm{m}$. The schematic and its equivalent-circuit model of the passive HEMT are shown in Fig. 1. The gate terminal is biased via a large resistor to prevent RF signal leakage. At $V_{g}=-3 \mathrm{~V}$, the off-state capacitor $\left(C_{\text {off }}\right)$ is $19 \mathrm{fF}$ with a parasitic resistor $\left(R_{p}\right)$ of $\left.5 \Omega\right)$, as shown in Fig. 1(a). At $V_{g}=0 \mathrm{~V}$, the on-state resistor $\left(R_{\mathrm{on}}\right)$ is $16 \Omega$ with a parasitic inductor $\left(L_{p}\right)$ of $20 \mathrm{pH}$, as shown in Fig. 1(b).

\section{ANALYSIS OF QUARTER-WAVELENGTH LOADED SIR}

SIRs have the advantages of miniature size and the control of higher order resonant frequencies [23], [24]. Fig. 2 shows the proposed circuit structure of a loaded quarter-wavelength SIR and its simplified equivalent circuits in different bias conditions. The passive HEMT $\left(Z_{L}\right)$ is loaded at the open end of the quarter-wavelength SIR. By applying different bias conditions to the gate terminal of the HEMT device, the $Z_{L}$ could be either a capacitive or an inductive load. For simplicity of the analysis, the parasitic resistors of the passive HEMT model are neglected. This assumption has been verified in a high-frequency switch design [11].

It is noted that, different from the half-wavelength structure in [17], the quarter-wavelength structure is short circuited at the unloaded end. Therefore, the resonant conditions of the loaded quarter-wavelength SIR in Fig. 2 can be obtained by setting
$Z_{\text {in }}=0$, where $Z_{\text {in }}$ is the input impedance of the SIR looking into the short end. The resonant condition could be derived as

$Z_{L}\left(Z_{01}-Z_{02} \tan \theta_{1} \tan \theta_{2}\right)+j Z_{01}\left(Z_{01} \tan \theta_{1}+Z_{02} \tan \theta_{2}\right)=0$

where $Z_{01}$ and $Z_{02}$ are the characteristic impedances of the two line sections, and $\theta_{1}$ and $\theta_{2}$ are the electrical lengths of the two line sections.

If the length ratio $\alpha$ of the SIR is defined as

$$
\alpha=\frac{\theta_{1}}{\theta_{1}+\theta_{2}}=\frac{\theta_{1}}{\theta_{t}}
$$

applying (2) into (1) yields

$$
\begin{aligned}
Z_{L}\left[Z_{01}-\right. & \left.Z_{02} \tan \alpha \theta_{t} \tan (1-\alpha) \theta_{t}\right] \\
& +j Z_{01}\left[Z_{01} \tan \alpha \theta_{t}+Z_{02} \tan (1-\alpha) \theta_{t}\right]=0 .
\end{aligned}
$$

It is known that the $Z_{L}$ could be either a capacitive or an inductive load in different bias conditions. When $V_{g}=-3 \mathrm{~V}$, the loaded resonator is terminated by a capacitive load. The capacitive load can be treated as a part of the open end, and thus, the resonator acts as a quarter-wavelength resonator. The resonant condition can then be determined by applying $Z_{L}=-j X_{C}$ into (3), which yields

$$
\begin{aligned}
Z_{02}\left[Z_{01} \tan (1-\alpha) \theta_{t}\right. & \left.+X_{C} \tan \left(\alpha \theta_{t}\right) \tan (1-\alpha) \theta_{t}\right] \\
& +Z_{01}\left[Z_{01} \tan \left(\alpha \theta_{t}\right)-X_{C}\right]=0 .
\end{aligned}
$$

When $V_{g}=0 \mathrm{~V}$, the passive HEMT becomes a inductive load. The inductive load can be considered as a part of the short end, and the resonant condition of the loaded resonator will behave as a half-wavelength resonator (both ends are short). The resonant condition can be obtained by substituting $Z_{L}=j X_{L}$ into (3), and it can be simplified as

$$
\begin{aligned}
Z_{02}\left[Z_{01} \tan (1-\alpha) \theta_{t}-\right. & \left.X_{L} \tan \left(\alpha \theta_{t}\right) \tan (1-\alpha) \theta_{t}\right] \\
& +Z_{01}\left[Z_{01} \tan \left(\alpha \theta_{t}\right)+X_{L}\right]=0 .
\end{aligned}
$$

For convenience in later discussions, we define the resonator loaded with a capacitive load as the on-mode resonator $\left(V_{g}=\right.$ $-3 \mathrm{~V}$ ) and the resonator loaded with an inductive load as the off-mode resonator $\left(V_{g}=0 \mathrm{~V}\right)$. The length ration $(\alpha)$ versus the ratio of second resonant frequency $\left(f_{1}\right)$ to the fundamental resonant frequency $\left(f_{0}\right)$ of the on-mode resonator are shown in Fig. 3(a), and length ration $(\alpha)$ versus the ratio of first resonant frequency of off-mode resonator $\left(f_{0}^{\prime}\right)$ to the fundamental resonant frequency $\left(f_{0}\right)$ of the on-mode resonator are shown in Fig. 3(b). The capacitive and inductive values in this figure are based on the device parameters given in Section II, and the fundamental frequency is the designed center frequency of $40 \mathrm{GHz}$. From Fig. 3 , it is observed that the ratios of $f_{1} / f_{0}$ swing around the center at approximately 3 , and the ratios of $f_{0}^{\prime} / f_{0}$ swing around the center at approximately 2.2 with respect to different impedance and length ratios. 


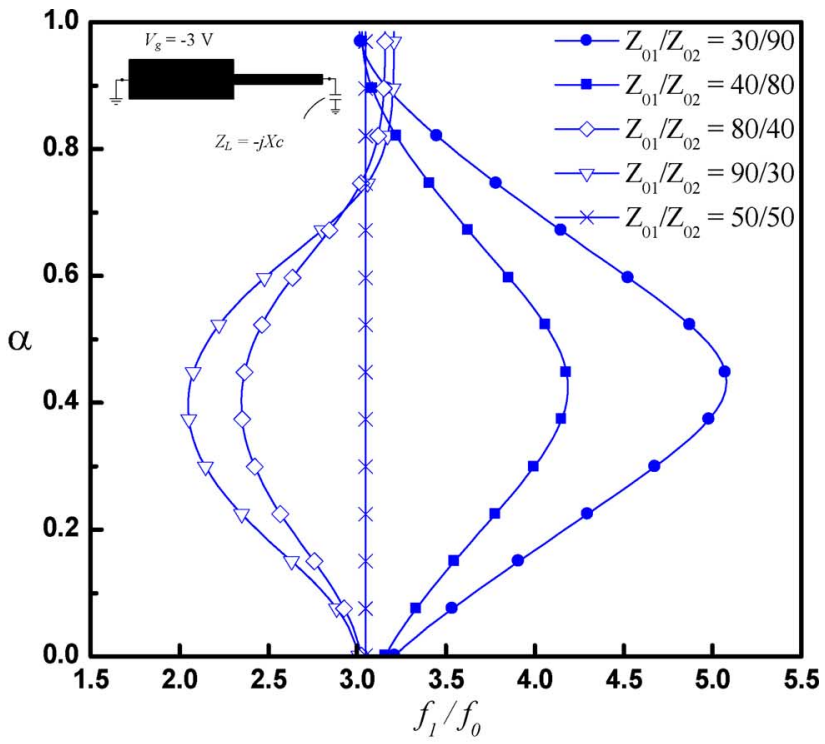

(a)

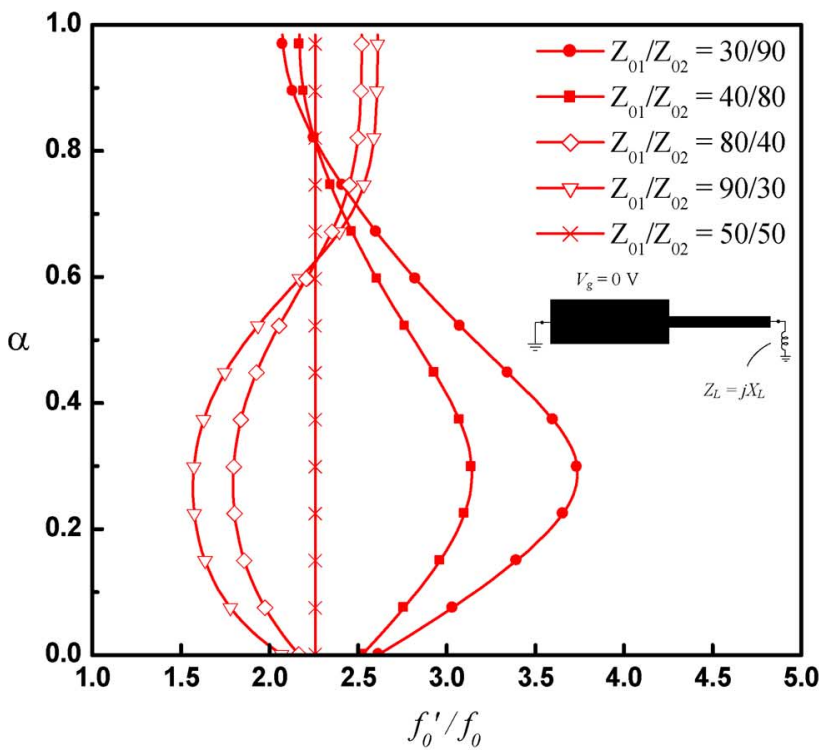

(b)

Fig. 3. Length ratio $(\alpha)$ versus resonant frequency ratio in different steppedimpedance conditions. (a) $f_{1} / f_{0}$, (b) $f_{0}^{\prime} / f_{0}$.

Compared with the half-wavelength loaded SIRs in [17], the frequency span between the on and off mode of a quarter-wavelength resonators is approximately twice that of a half-wavelength resonator since a quarter-wavelength resonator only resonates at odd modes. Moreover, taking advantage of the stepped-impedance characteristics, the frequency span of each mode can be further apart. This feature facilitates the goal of wide separation of the undesired resonant frequencies in this design. In addition to the unwanted resonant frequency separations, the quarter-wavelength resonators also effectively reduces the overall circuit size. Theoretically, the occupied area of a quarter-wavelength resonator is only one-quarter of a half-wavelength resonator. This is attractive for size reduction in MMIC designs.

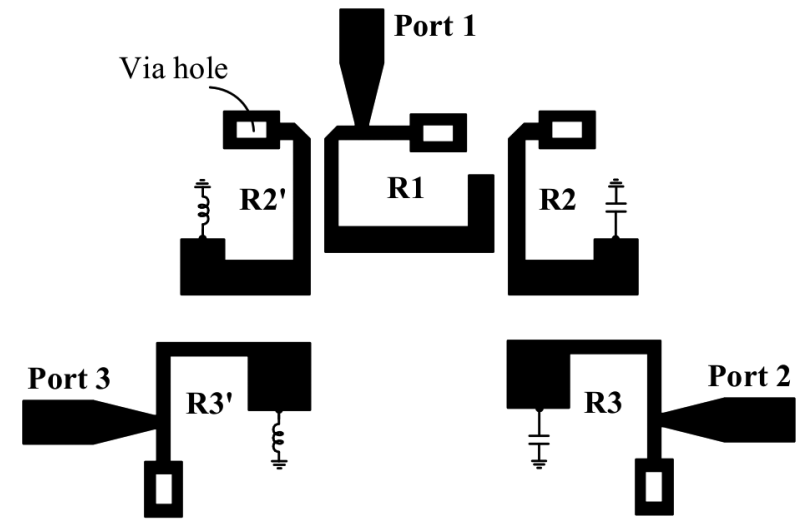

(a)

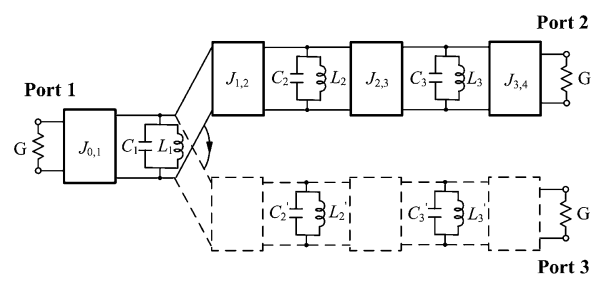

(b)

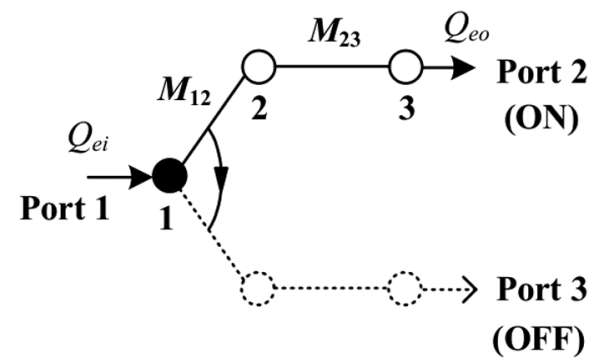

(c)

Fig. 4. (a) Circuit schematic, (b) equivalent circuit, and (c) coupling structure of the third-order SPDT filter integrated switch.

\section{40-GHz MMIC SPDT FILTER-INTEGRATED SWITCH}

The SPDT filter-integrated switch is designed to have the function of an SPDT switch and a third-order bandpass filter. In the on state, the loaded passive HEMTs are turned off; the loaded SIRs behave as quarter-wavelength resonators. The loaded and unloaded resonators resonate at the same fundamental frequency, but at different higher order resonant frequencies to form a coupled-resonator bandpass filter with spurious suppression. In the off state, the loaded passive HEMTs are turned on; the loaded SIRs are switched to act as half-wavelength resonators. With the selection of proper impedance and length ratio of each SIR, the resonant frequencies of the loaded SIRs and unloaded SIRs between input and output ports would be staggered over the band of interest to achieve a good wideband isolation. Therefore, the proposed filter-integrated switch can perform a bandpass function with wide stopband rejection and a wideband isolation in its on and off states.

Fig. 4 shows the circuit schematic and equivalent circuit of the proposed third-order SPDT bandpass filter integrated switch. In 
Fig. 4(b), the admittance inverter is used to represent the coupling between resonators, where $J$ denotes the characteristic admittance [25]. In Fig. 4(a), there is one unloaded resonator and two loaded resonators between each input and output port. Using (4), (5), and their corresponding figure (Fig. 3), the unloaded resonator R1 and the on-mode resonators are designed to have the same fundamental frequency, while with different higher order resonant frequencies, and the resonant frequencies of the off-mode resonators and R1 are misaligned over the band of interest. Fig. 4(c) shows the proposed coupling structure of the SPDT circuit, where the solid dot represents a unloaded resonator, each hollow circle represents an HEMT loaded resonator, and the solid lines are the main path couplings. In the proposed coupling structure, the first resonator R1 plays the role as a shared resonator, which is used to combine two SPST filter integrated circuits into a SPDT filter integrated switch. The use of shared resonator can not only save the area for a matching T-junction needed in a conventional switch design [8]-[12], but also reduces the total number of resonators. As a consequence, the total circuit size could be significantly reduced.

In this SPDT circuit [see Fig. 4(a)], the loaded resonators R2, $\mathrm{R} 2^{\prime}, \mathrm{R} 3$, and $\mathrm{R} 3^{\prime}$ are used to control the RF signal from port 1 to ports 2 or 3. Considering the condition in Fig. 4(a), R2-R3 are switched to on-mode resonators $\left(V_{g}=-3 \mathrm{~V}\right)$ and $\mathrm{R} 2^{\prime}-\mathrm{R} 3^{\prime}$ are switched to off-mode resonators $\left(V_{g}=0 \mathrm{~V}\right)$. With the specific coupling coefficient between each resonator and external quality factor of input/out resonator, a third-order bandpass filter composed by R1, R2, and R3 is formed. Since resonators R1, $\mathrm{R} 2$, and R3 have different higher order resonant frequency, the spurious response of the synthesized filter can be suppressed. At the same time, the first resonant frequencies of the off-mode resonator $\mathrm{R} 2^{\prime}$ and $\mathrm{R} 3^{\prime}$ are distributed over approximately twice of the center frequency. The resonant frequencies of R1, R2 and $\mathrm{R} 3^{\prime}$, are misaligned over a wide frequency range, therefore, no passband response will appear at port 3 . As a result, a wideband isolation state can be obtained at port 3 . It is noted that the passband will not be affected in the presence of resonators $\mathrm{R} 2^{\prime}$ and $\mathrm{R} 3^{\prime}$ because the resonant frequencies of the off-state resonators are not in the vicinity of the center frequency. In this case, the RF signal will pass from ports 1 to 2 at a center frequency of $40 \mathrm{GHz}$ with port 3 in the isolation state. If the bias condition is reversed, the passband signal will pass through port 3 with port 2 in the isolation state. In this SPDT filter-integrated switch, the two SPST filter-integrated switches can be designed independently, and the two SPST circuits are combined via the common resonator R1 to form the SPDT bandpass filter-integrated switch.

The resonant frequencies of the loaded and unloaded SIRs in Fig. 4(a) are listed in Table I. It can be observed that, in the on-state, each resonator has the same fundamental resonant frequency of $40 \mathrm{GHz}$ and different higher order resonant frequencies. In the off state, the resonant frequencies of the resonators in the isolated path are misaligned.

The third-order filter integrated switch was designed to have the specifications of a Butterworth response at the center frequency of $40 \mathrm{GHz}$, and 3-dB fractional bandwidth $\left(\Delta_{3} \mathrm{~dB}\right)$ of $8 \%$. The lumped circuit element values of the low-pass prototype filter are $g_{0}=1, g_{1}=1, g_{2}=2, g_{3}=1$, and $g_{4}=1$.
TABLE I

RESONANT FREQUENCIES OF EACH RESONATOR AT ON AND OFF STATES

\begin{tabular}{lccccc}
\hline & \multicolumn{2}{c}{ ON-state } & & \multicolumn{2}{c}{ OFF-state } \\
\cline { 2 - 3 } \cline { 5 - 6 } & $f_{0}(\mathrm{GHz})$ & $f_{1}(\mathrm{GHz})$ & & $f_{0}(\mathrm{GHz})$ & $f_{1}(\mathrm{GHz})$ \\
\hline \hline R1 & 40 & 113 & & 40 & 113 \\
R2 & 40 & 103 & & 87 & 170 \\
R3 & 40 & 107 & & 94 & 163 \\
\hline
\end{tabular}

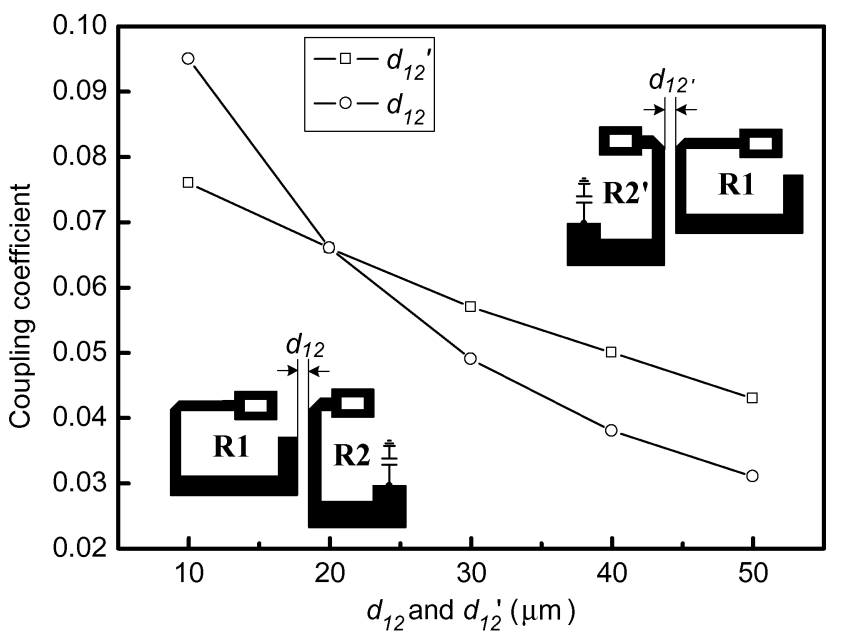

(a)

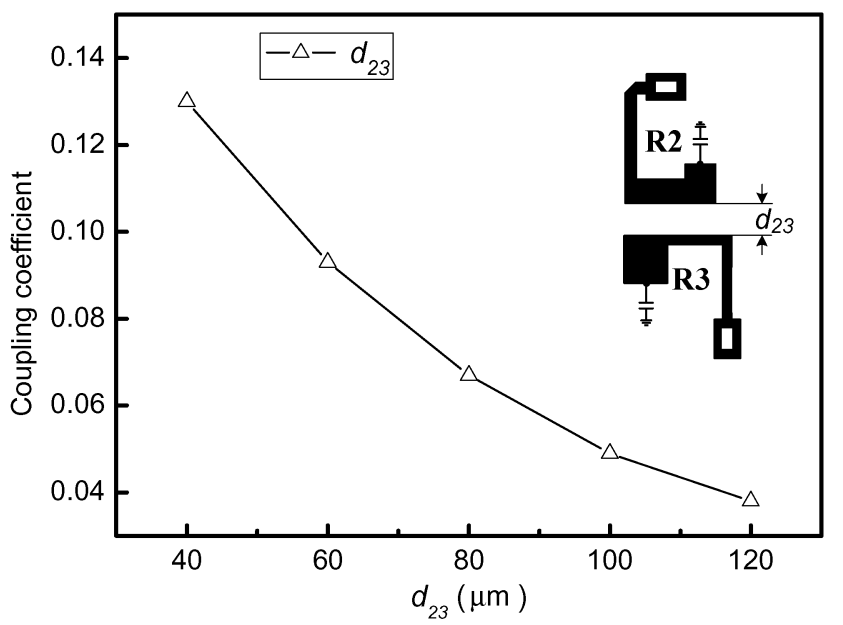

(b)

Fig. 5. Coupling structures and design curves for: (a) $M_{12}$ and (b) $M_{23}$.

Thus, the coupling coefficients and the external quality factor $Q_{\text {ext }}$ can be obtained as

$$
\begin{aligned}
& M_{12}=\frac{\Delta_{3 \mathrm{~dB}}}{\sqrt{g_{1} g_{2}}}=\frac{\Delta_{3 \mathrm{~dB}}}{\sqrt{g_{2} g_{3}}}=M_{23}=0.056 \\
& Q_{e i}=\frac{g_{0} g_{1}}{\Delta_{3 \mathrm{~dB}}}=\frac{g_{3} g_{4}}{\Delta_{3 \mathrm{~dB}}}=Q_{e o}=12.5
\end{aligned}
$$

where $M_{j k}$ represents the coupling coefficient between resonators $j$ and $k(j, k=1,2,3, \ldots)$, and $Q_{e i}$ and $Q_{e o}$ are the external quality factors associated with the input and output couplings, respectively [25]. 


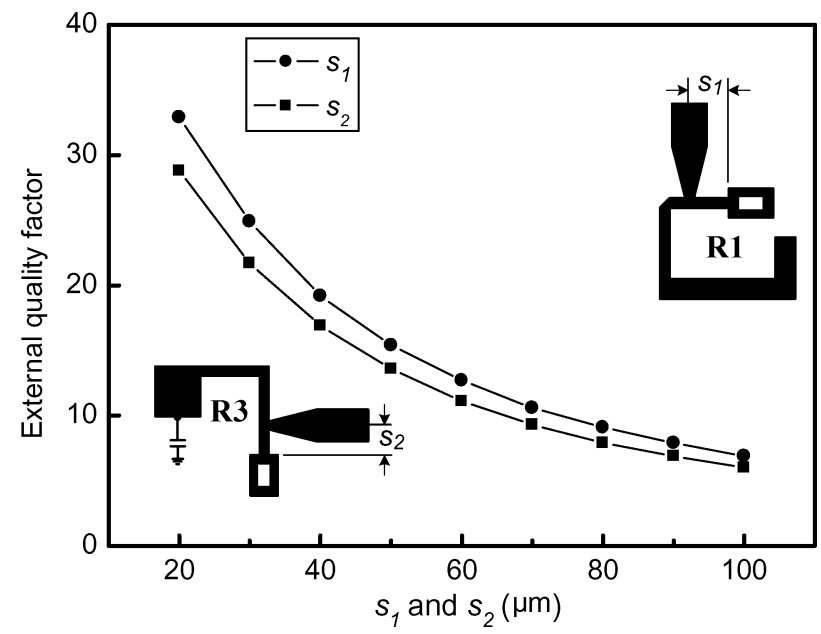

Fig. 6. Design curves of input and output external quality factors.

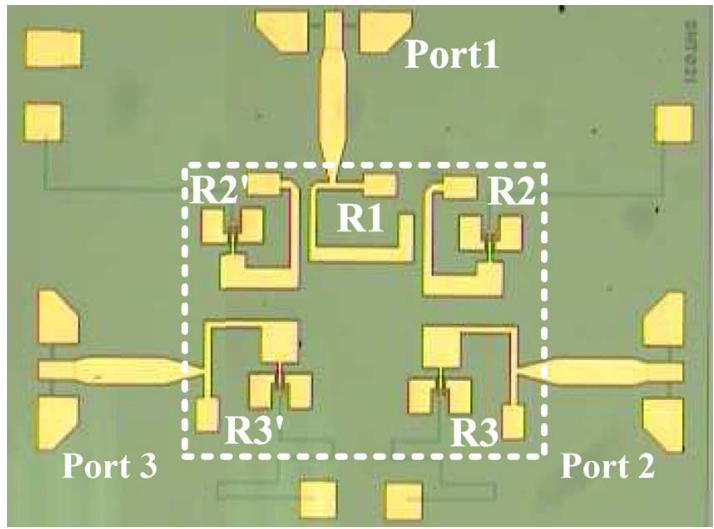

Fig. 7. Chip photograph of the SPDT filter integrated switch, and the chip size is $2 \times 1.5 \mathrm{~mm}^{2}$.

Fig. 5(a) shows the design curves for determining the distance between $\mathrm{R} 1$ and $\mathrm{R} 2\left(\mathrm{R} 2^{\prime}\right)$, and the curve for determining the distance between R2 and R3 is shown in Fig. 5(b). Fig. 6 shows the simulated external quality factors versus the tapped line position of resonators 1 and 3 , which are used to decide the tapped position of the I/O ports. These results are extracted by using the electromagnetic (EM) software Sonnet [26].

With the parameters given by (6), the physical distances can be obtained from Figs. 5 and 6. Geometric parameters of $d_{12}=$ $27 \mu \mathrm{m}, d_{12}^{\prime}=32 \mu \mathrm{m}, d_{23}=92 \mu \mathrm{m}, s_{1}=61 \mu \mathrm{m}$, and $s_{2}=$ $55 \mu \mathrm{m}$ were used.

Fig. 7 shows a photograph of the SPDT filter integrated switch. The total chip size is $2 \times 1.5 \mathrm{~mm}^{2}$, which is to comply with the dicing requirement in the multiproject wafer (MPW). The effective circuit area is only $950 \times 760 \mu \mathrm{m}^{2}$ $\left(0.37 \lambda_{0} \times 0.3 \lambda_{0}\right)$, as indicated via the dotted lines, where $\lambda_{0}$ is the guided wavelength of microstrip line on this substrate at the center frequency.

The circuit was tested via on-wafer probing. Fig. 8 shows the measured $S_{11}, S_{21}$, and $S_{31}$ in the on and off states of the circuit. During testing, only one port was set at the thru state, while the other output port was at the isolation state. In Fig. 8(a), at the thru port (port 2), a measured bandpass response with a passband insertion loss of $3.1 \mathrm{~dB}$ with an input return loss of $15 \mathrm{~dB}$

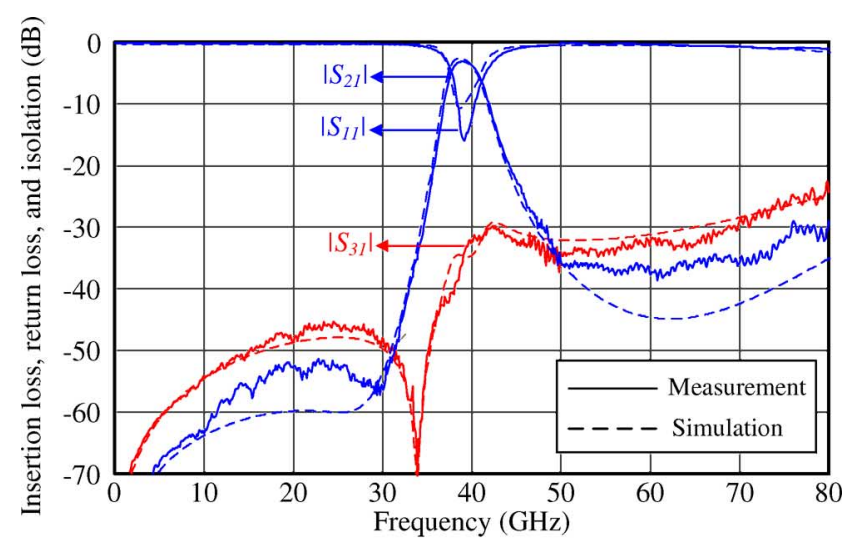

(a)

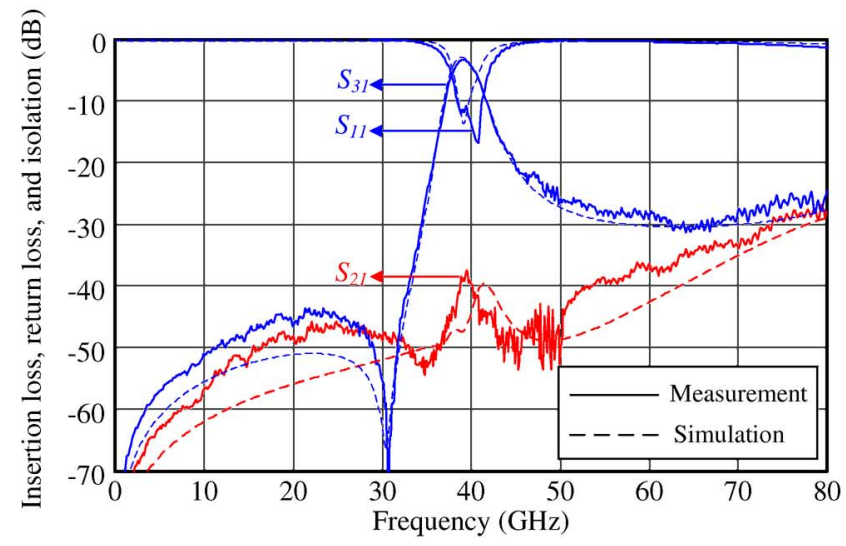

(b)

Fig. 8. $S$-parameters measurement results of the filter integrated switch. (a) Port 2 is on and port 3 is off. (b) Port 3 is on and port 2 is off.

at a center frequency of $39 \mathrm{GHz}$ were plotted against simulations, and the measured $3-\mathrm{dB}$ fractional bandwidth is $8 \%$. At the isolation port (port 3), the circuit shows a measured isolation greater than $30 \mathrm{~dB}$ at the center frequency, and a wideband isolation better than $30 \mathrm{~dB}$ from dc to $69 \mathrm{GHz}$. The measured results agree well with the simulations. Fig. 8(b) shows the simulated and measured results when port 3 is turned on and port 2 is turned off. The measured passband insertion loss is $3.2 \mathrm{~dB}$ with an input return loss of $11 \mathrm{~dB}$ at $39 \mathrm{GHz}$. The isolation at port 2 is approximately $38 \mathrm{~dB}$ at the center frequency, and a wideband isolation of better than $35 \mathrm{~dB}$ from de to $64 \mathrm{GHz}$ was also observed. Fig. 9 shows the measured results of the output power versus input power at the thru port, and the input $P_{1 \mathrm{~dB}}$ is approximately $15 \mathrm{dBm}$.

\section{V. $40-\mathrm{GHz}$ MMIC MULTIPLE-PORT FILTER-INTEGRATED SWITCH}

Multiple-port switches have been published and realized in many processes such as GaAs, CMOS, and microelectromechanical systems (MEMS) [19]-[22]. In designing a millimeterwave multiple-ports switch, matching cross-junctions are usually needed to connect I/O arms. However, these cross-junctions occupy large chip areas and increase the difficulty in the layout. Since the cross-junctions are usually formed by quarter-wavelength transmission lines, the loading effect of the I/O arms 


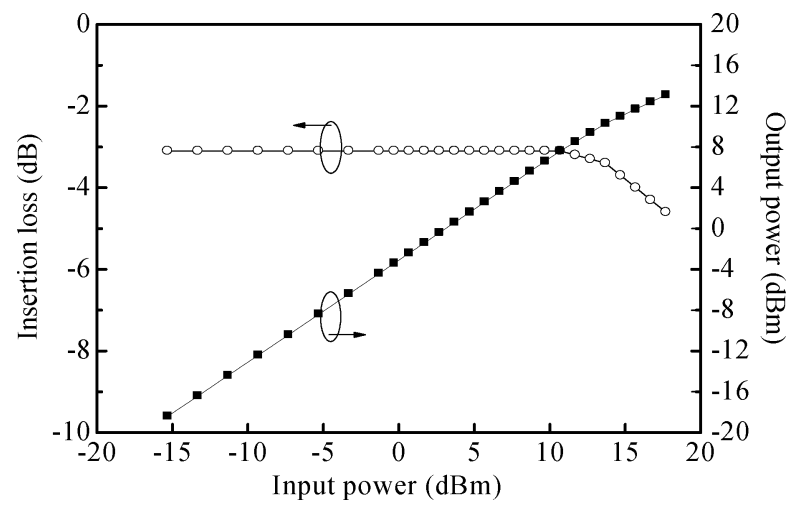

Fig. 9. Measured output power versus input power at the center frequency of the SPDT filter-integrated switch.

has always been an issue in the millimeter-wave multiple-port switch design.

In this multiple-port filter-integrated switch design, the shared resonator is used to replace the cross-junctions, which are used to combine the $\mathrm{I} / \mathrm{O}$ arms in conventional switch designs [8]-[12]. Consequently, there will be no loading effects from the matching cross-junctions in this multiple-port filter-integrated switch. Therefore, this circuit provides another solution in designing a millimeter-wave multiple-port switch. Moreover, the shared resonator technique can lower the total number of resonators. Hence, a significant chip size reduction could be achieved.

Fig. 10 shows the circuit schematic and proposed coupling structure of a fourth-order six-port bandpass filter-integrated switch. In Fig. 10(a), the loaded SIRs are designed to have the same fundamental frequency of $40 \mathrm{GHz}$ with different higher order spurious frequencies in the on mode and staggered resonant frequencies in the off mode. Unlike an SPDT filter-integrated switch, the difficulty of realizing this filter-integrated switch is that there are six ports (two for input ports and four for output ports) in this circuit. In order to increase coupling periphery of the shared resonator $\mathrm{R} 2$, a half-wavelength resonator structure is chosen. The fundamental frequency of R2 is also designed at $40 \mathrm{GHz}$. Fig. 10(b) shows the coupling structure of this multiple-port filter-integrated switch. The shared resonator R2 plays the role as a bridge to pass the RF signal from input ports to output ports. For example, when the switchable resonators $\mathrm{R} 1, \mathrm{R} 3$, and R4 are switched to the on mode, the others are switched to the off mode. According to the coupled-resonator filter theory, the RF signal of the passband frequency will pass from input port 1 via resonator R1-R2-R3-R4, and then to the output port 3. Similarly, the RF signal flow can be controlled from one of the two input ports to one of the four output ports.

In this six-port filter-integrated switch, by taking advantage of the shared resonator R2, there is no need for the matching junction. As a consequence, the total circuit size is significantly reduced. Each SPST filter-integrated switch could be designed independently, and the six-port filter-integrated switch is then formed by integrating the SPST ones via the common resonator R2.

The fourth-order filter integrated switch was designed to have the specifications of a Chebyshev response at the center

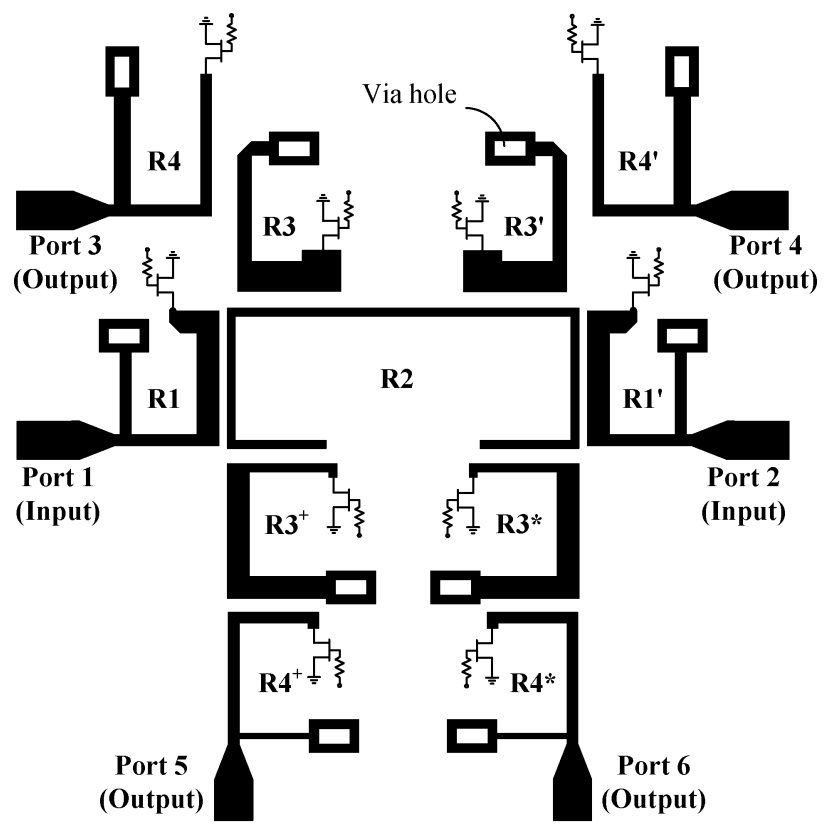

(a)

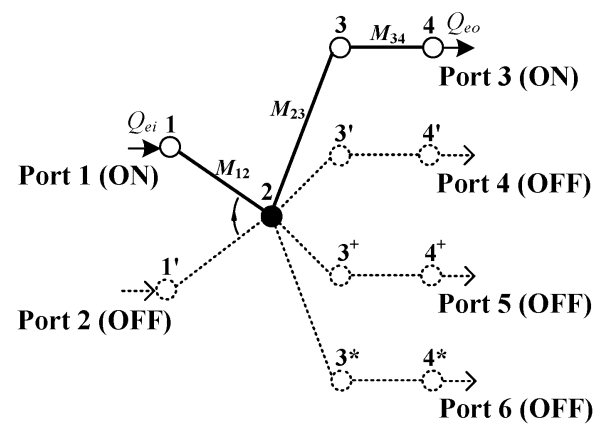

(b)

Fig. 10. (a) Circuit schematic and (b) equivalent circuit of the fourth-order sixport filter-integrated switch.

frequency of $40 \mathrm{GHz}$ with 3-dB fractional bandwidth $\left(\Delta_{3} \mathrm{~dB}\right)$ of $13 \%$. From (6), the required coupling matrix and external quality factor are listed as follows:

$$
\begin{aligned}
{[M] } & =\left[\begin{array}{cccc}
0 & 0.108 & 0 & 0 \\
0.108 & 0 & 0.085 & 0 \\
0 & 0.085 & 0 & 0.108 \\
0 & 0 & 0.108 & 0
\end{array}\right] \\
Q_{e i} & =Q_{e o}=8.5 .
\end{aligned}
$$

With the given design parameters, following the same design procedure of the SPDT filter-integrated switch discussed in Section IV, the geometry parameters can be determined.

Fig. 11 shows the photograph of the six-port filter integrated switch. The total chip size is $2 \times 2 \mathrm{~mm}^{2}$. The effective circuit area is $1215 \times 1500 \mu \mathrm{m}^{2}\left(0.47 \lambda_{0} \times 0.58 \lambda_{0}\right)$, as indicated by the dotted lines, where $\lambda_{0}$ is the guided wavelength of the microstrip line on this substrate at the center frequency. It is noted that for testing purposes, there are two output ports terminated with on-chip $50 \Omega$ thin-film resistors.

During testing, when the resonators between ports 1 and 3 (R1, R2, R3, and R4) are switched to the on mode $\left(V_{g}=-3 \mathrm{~V}\right)$, while the others are switched to the off mode $\left(V_{g}=0 \mathrm{~V}\right)$. The 


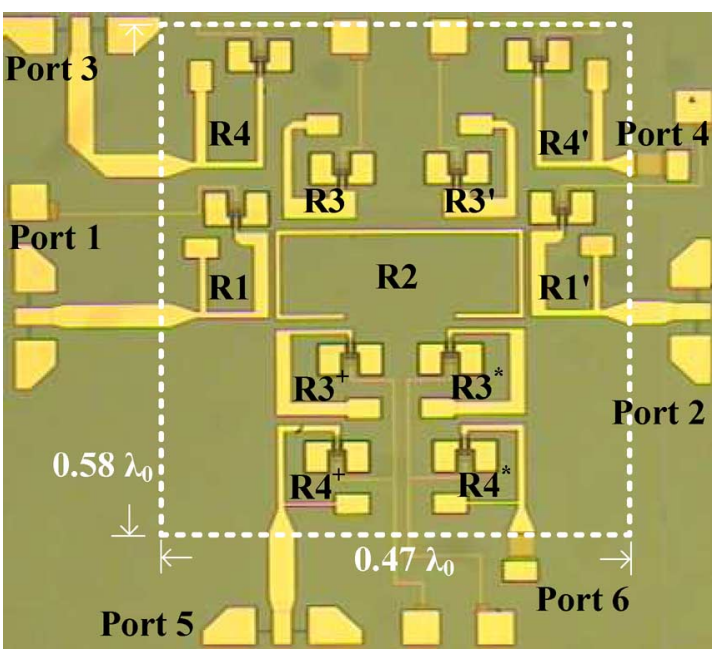

Fig. 11. Chip photograph of the 40-GHz six-port filter-integrated switch, and the chip size is $2 \times 2 \mathrm{~mm}^{2}$.

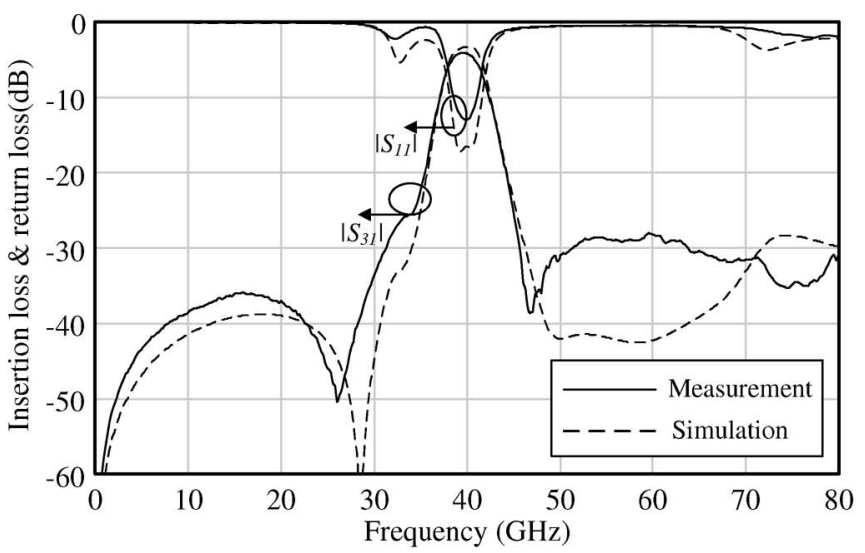

(a)

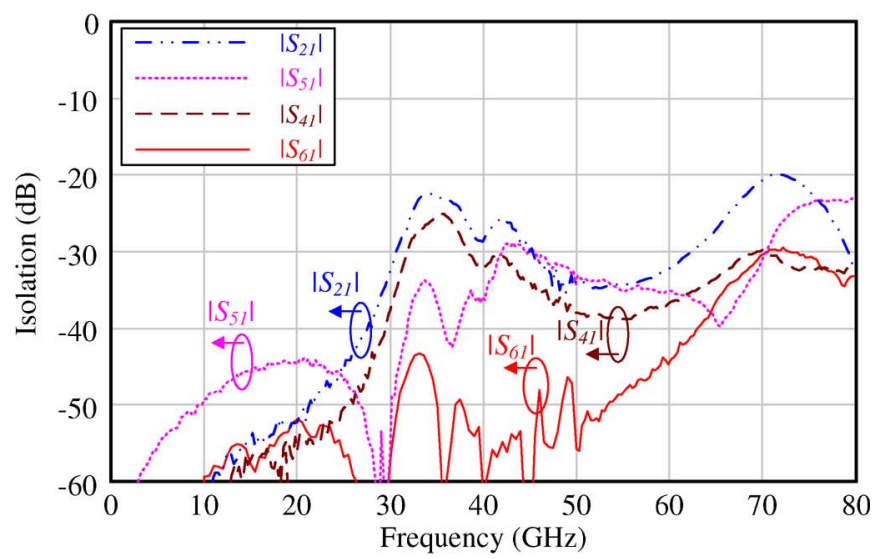

(b)

Fig. 12. Measured results of the six-port filter-integrated switch when ports 1 and 3 are ON. (a) Results at the thru ports. (b) Results at the isolation ports.

RF signal will pass from ports 1 to 3 . Fig. 12 shows the measured results when ports 1 and 3 are ON, and the other ports are OFF. Fig. 12(a) shows the simulated and measured results of the thru ports, a bandpass response with a passband insertion loss of $3.9 \mathrm{~dB}$ was measured at the center frequency of $40 \mathrm{GHz}$. At the other isolation ports, the measured isolations are all greater

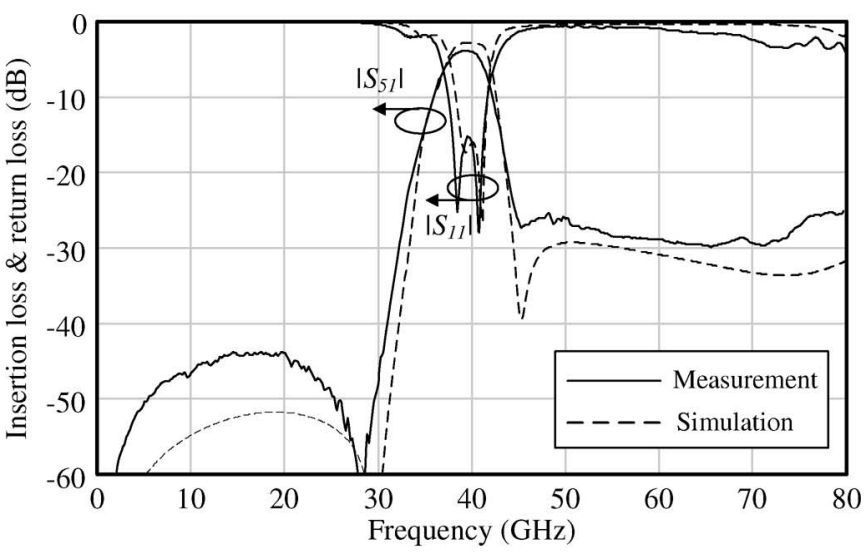

(a)

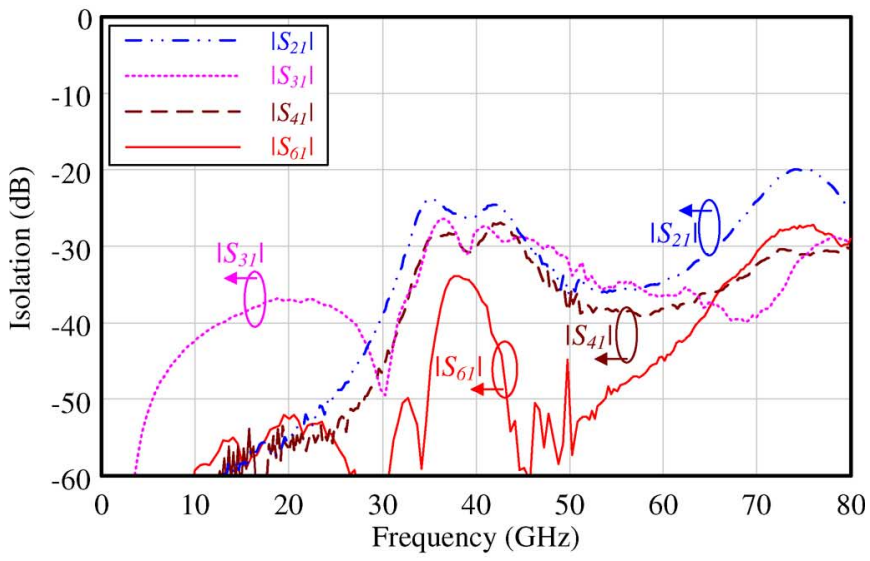

(b)

Fig. 13. Measured results of the six-port filter-integrated switch when ports 1 and 5 are ON. (a) Results at the thru ports. (b) Results at the isolation ports.

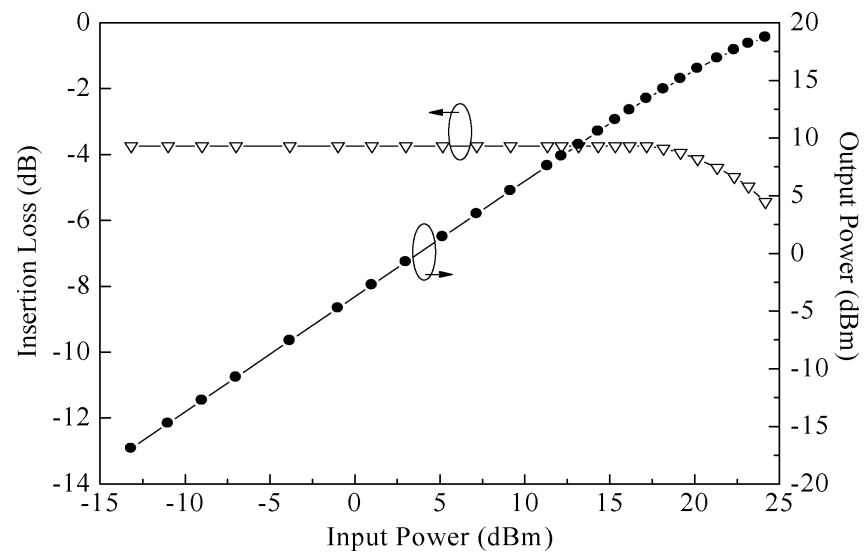

Fig. 14. Measured output power versus input power at $40 \mathrm{GHz}$ of the six-port filter-integrated switch in the on state $\left(S_{31}\right)$.

than $27 \mathrm{~dB}$ at the center frequency, as shown is Fig. 12(b). Considering another testing condition, ports 1 and 5 are ON, and the other ports are OFF. Fig. 13(a) shows the measured and simulated results at the thru ports. A bandpass response with a passband insertion loss of $3.7 \mathrm{~dB}$ was measured at a center frequency of $40 \mathrm{GHz}$. At the other isolation ports, the measured isolations are all greater than $25 \mathrm{~dB}$ at the center frequency, as shown is Fig. 13(b). Fig. 14 shows the measured results of the output 
power versus input power at the thru port $\left(S_{31}\right)$, and the input $P_{1 \mathrm{~dB}}$ is approximately $17 \mathrm{dBm}$.

\section{CONCLUSION}

In this paper, compact $40-\mathrm{GHz}$ MMIC SPDT and multiple-port bandpass filter-integrated switches have been proposed. The bandpass filter-integrated switches are designed based on the switchable resonators to have bandpass response in the on state and wideband isolation in the off state. The HEMT loaded at the open end of the quarter-wavelength SIR is developed as a switchable resonator, which is used to switch the resonant frequency and control the RF signal flow. Moreover, in order to compact the overall chip size, the concept of shared resonator is also used to lower the number of resonators. The proposed circuits can indeed increase the level of RF transceiver integration.

\section{ACKNOWLEDGMENT}

The authors would like to thank Prof. G. Vendelin, National Central University, JhongLi, Taiwan, R.O.C., for his valuable suggestions.

The chip was fabricated by WIN Semiconductors through the Chip Implementation Center (CIC), Taiwan, R.O.C.

\section{REFERENCES}

[1] M. J. Roberts, S. Iezekiel, and C. M. Snowden, "A $W$-band self-oscillating subharmonic MMIC mixer," IEEE Trans. Microw. Theory Tech., vol. 46, no. 12, pp. 2104-2018, Dec. 1998.

[2] J. R. Long, "A low-voltage 5.1-5.8-GHz image-reject downconverter RF IC," IEEE J. Solid-State Circuits, vol. 35, no. 9, pp. 1320-1328, Sep. 2003.

[3] I.-J. Chen, H. Wang, and P. Hsu, "A $V$-band quasi-optical GaAs HEMT monolithic integrated antenna and receiver front end," IEEE Trans. Microw. Theory Tech., vol. 51, no. 12, pp. 2461-2468, Dec. 2003.

[4] T. P. Wang, C.-C. Chang, R.-C. Liu, M.-D. Tsai, K.-J. Sun, Y.-T. Chang, L.-H. Lu, and H. Wang, "A low-power oscillator mixer in 0.18$\mu \mathrm{m}$ CMOS technology," IEEE Trans. Microw. Theory Tech., vol. 54, no. 1, pp. 88-95, Jan. 2006.

[5] F. J. Huang and K. O, "A 0.5- $\mu \mathrm{m}$ CMOS T/R switch for $900-\mathrm{MHz}$ wireless applications," IEEE J. Solid-State Circuits, vol. 36, no. 3, pp. 486-492, Mar. 2001.

[6] C. Tinella, J. M. Fournier, D. Belot, and V. Knopik, "A high-performance CMOS-SOI antenna switch for the 2.5-5-GHz band," IEEE J. Solid-State Circuits, vol. 38, no. 7, pp. 1279-1283, Jul. 2003.

[7] Z. Li and K. K. O, "15-GHz fully integrated nMOS switches in a 0.13- $\mu$ m CMOS process," IEEE J. Solid-State Circuits, vol. 40, no. 11, pp. 2323-2328, Nov. 2005.

[8] J. Kim, W. Ko, S. H. Kim, J. Jeong, and Y. Kwon, "A high-performance 40-85 GHz MMIC SPDT switch using FET-integrated transmission line structure," IEEE Microw. Wireless Compon. Lett., vol. 13, no. 12, pp. 505-507, Dec. 2003.

[9] K. Y. Lin, W. H. Tu, P. Y. Chen, H. Chen, H. Wang, and R. B. Wu, "Millimeter-wave MMIC passive HEMT switches using traveling-wave concept," IEEE Trans. Microw. Theory Tech., vol. 52, no. 8, pp. 1798-1808, Aug. 2004.

[10] M. C. Yeh, Z. M. Tsai, H. Wang, C. Y. Su, and C. P. Chao, "A millimeter-wave wideband SPDT switch with traveling-wave concept using 0.13- $\mu \mathrm{m}$ CMOS process," in IEEE MTT-S Int. Microw. Symp. Dig., Jun. 2005, [CR ROM].

[11] Z. M. Tsai, M. C. Yeh, M. F. Lei, H. Y. Chang, C. S. Lin, and H. Wang, "FET-integrated CPW and the application in filter synthesis design method on traveling-wave switch above $100 \mathrm{GHz}$," IEEE Trans. Microw. Theory Tech., vol. 54, no. 5, pp. 2090-2097, May 2006.
[12] S.-F. Chao, H. Wang, C.-Y. Su, and J. G. J. Chern, "A 50-94 GHz CMOS SPDT switch using traveling-wave concept," IEEE Microw. Wireless Compon. Lett., vol. 17, no. 2, pp. 130-132, Feb. 2007.

[13] T. S. Martin, F. Wang, and K. Chang, "Theoretical and experimental investigation of novel varactor-tuned switchable microstrip ring resonator circuits," IEEE Trans. Microw. Theory Tech., vol. 36, no. 12, pp. 1733-1739, Dec. 1988.

[14] Y. H. Shu, J. A. Navarro, and K. Chang, "Electronically switchable and tunable coplanar waveguide-slotline bandpass filters," IEEE Trans. Microw. Theory Tech., vol. 39, no. 3, pp. 548-554, Mar. 1991.

[15] S. F. Chang, W.-L. Chen, J.-L. Chen, H.-W. Kung, and H.-Z. Hsu, "New millimeter-wave MMIC switch design using the image-filter synthesis method," IEEE Microw. Wireless Compon. Lett., vol. 14, no. 3 , pp. 103-105, Mar. 2004.

[16] Z.-M. Tsai, Y.-S. Jiang, J. Lee, K.-Y. Lin, and H. Wang, "Bandpass single-pole-double-throw FET quarter-wavelength bandpass filter-integrated-switch," IEEE Trans. Microw. Theory Tech., vol. 55, no. 8, pp. 1601-1610, Aug. 2007.

[17] S.-F. Chao, C.-H. Wu, Z.-M. Tsai, H. Wang, and C.-H. Chen, "Electronically switchable bandpass filters using loaded stepped-impedance resonators," IEEE Trans. Microw. Theory Tech., vol. 54, no. 12, pp. 4193-4201, Dec. 2006.

[18] S.-F. Chao, C.-C. Kuo, Z.-M. Tsai, and H. Wang, "A 40-GHz MMIC SPDT bandpass filter integrated switch," in IEEE MTT-S Int. Microw. Symp. Dig., Jun. 2007, pp. 483-486.

[19] G.-L. Tan, R. E. Mihailovich, J. B. Hacker, J. F. DeNatale, and G. M. Rebeiz, "Low-loss 2- and 4-bit TTD MEMS phase shifters based on SP4T switches," IEEE Trans. Microw. Theory Tech., vol. 51, no. 1, pp. 297-304, Jan. 2003.

[20] J. Lee, C. H. Je, S. Kang, and C.-A. Choi, "A low-loss single-pole six-throw switch based on compact RF MEMS switches," IEEE Trans. Microw. Theory Tech., vol. 53, no. 11, pp. 3335-3344, Nov. 2005.

[21] S.-F. Chao, Z.-M. Tsai, K.-Y. Lin, and H. Wang, "Compact $W$-band SPQT switch using traveling wave concept," in Proc. Eur. GAAS Conf., Paris, France, Oct. 2005, pp. 357-360.

[22] K. Jung and K. K. O, "A CMOS single-pole-four-throw switch," IEEE Microw. Wireless Compon. Lett., vol. 16, no. 3, pp. 128-130, Mar. 2006.

[23] M. Makimoto and S. Yamashita, "Bandpass filters using parallel coupled stripline stepped impedance resonators," IEEE Trans. Microw. Theory Tech., vol. MTT-28, no. 12, pp. 1413-1417, Dec. 1980.

[24] C.-W. Tang and S.-F. You, "Miniaturized wide stopband rejected microstrip filter with coupled spur-lines," Electron. Lett., vol. 42, no. 5, pp. 286-288, Mar. 2006.

[25] J. S. Hong and M. J. Lancaster, Microstrip Filter for RF/Microwave Applications. New York: Wiley, 2001, ch. 8.

[26] “Sonnet User's Manual, Release 10.0.," Sonnet Software Inc., North Syracuse, NY, 2004.

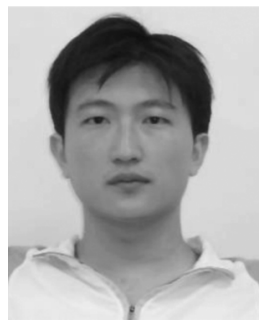

Shih-Fong Chao (S'05) was born in ChangHua, Taiwan, R.O.C., in 1979. He received the B.S. degree in electrical engineering from National Cheng-Kung University, Tainan, Taiwan, R.O.C., in 2002, and the $\mathrm{Ph} . \mathrm{D}$. degree in communication engineering from National Taiwan University, Taipei, Taiwan, R.O.C., in 2007.

His research interests include microwave and millimeter-wave circuit designs.

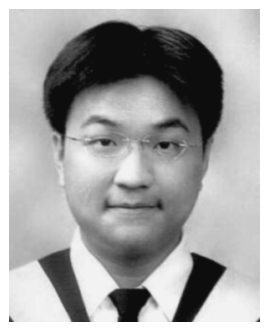

Che-Chung Kuo was born in Taipei, Taiwan, R.O.C., in 1980. He received the M.S. degrees in electrical engineering from the National Center University, Chung-Li, Taiwan, R.O.C., in 2005, and is currently working toward the Ph.D. degree in communication engineering at National Taiwan University, Taipei, Taiwan, R.O.C.

His research interests include microwave circuit designs and microwave circuit system-on-package integrations. 


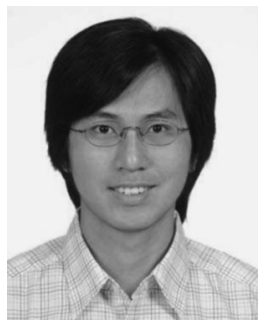

Zou-Min Tsai (S'00) was born in Mailo, Taiwan, R.O.C., in 1979. He received the B.S. degree in electronic engineering and Ph.D. degree in communication engineering from National Taiwan University, Taipei, Taiwan, R.O.C., in 2001 and 2006, respectively.

He is currently a Post-Doctoral Research Fellow with the Graduate Institute of Communication Engineering, National Taiwan University. His research interests are the theory of microwave or millimeterwave circuits.

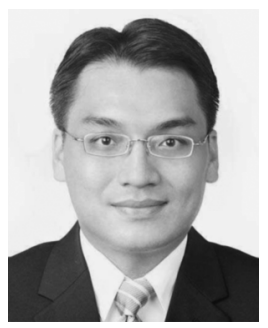

Kun-You Lin (S'00-M'04) was born in Taipei, Taiwan, R.O.C., in 1975. He received the B.S. degree in communication engineering from National Chiao Tung University, Hsinchu, Taiwan, R.O.C., in 1998, and the Ph.D. degree in communication engineering from National Taiwan University, Taipei, Taiwan, R.O.C., in 2003.

From August 2003 to March 2005, he was a Post-Doctoral Research Fellow with the Graduate Institute of Communication Engineering, National Taiwan University. From May 2005 to July 2006, he was an Advanced Engineer with the Sunplus Technology Company Ltd., Hsin-Chu, Taiwan, R.O.C. In July 2006, he joined the faculty of the Department of Electrical Engineering and Graduate Institute of Communication Engineering, National Taiwan University, as an Assistant Professor. His research interests include the design and analysis of microwave/RF circuits.

Dr. Lin is a member of Phi Tau Phi.

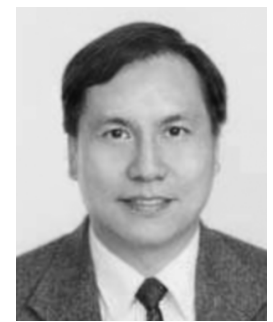

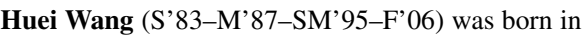
Tainan, Taiwan, R.O.C., on March 9, 1958. He received the B.S. degree in electrical engineering from National Taiwan University, Taipei, Taiwan, R.O.C., in 1980, and the M.S. and Ph.D. degrees in electrical engineering from Michigan State University, East Lansing, in 1984 and 1987, respectively.

During his graduate study, he was engaged in research on theoretical and numerical analysis of EM radiation and scattering problems. He was also involved in the development of microwave remote detecting/sensing systems. In 1987, he joined the Electronic Systems and Technology Division, TRW Inc. He has been an MTS and Staff Engineer responsible for MMIC modeling of computer-aided design (CAD) tools, MMIC testing evaluation, and design and became the Senior Section Manager of the Millimeter-Wave (MMW) Sensor Product Section, RF Product Center. In 1993, he visited the Institute of Electronics, National Chiao-Tung University, Hsinchu, Taiwan, R.O.C., to teach MMIC related topics. In 1994, he returned to TRW Inc. In February 1998, he joined the faculty of the Department of Electrical Engineering, National Taiwan University, as a Professor. He is currently the Director of the Graduate Institute of Communication Engineering, National Taiwan University.

Dr. Wang is a member of Phi Kappa Phi and Tau Beta Pi. He was the recipient of the Distinguished Research Award of National Science Council, R.O.C. (2003-2006). In 2005, he was elected as the first Richard M. Hong Endowed Chair Professor of National Taiwan University. He has been appointed an IEEE Distinguished Microwave Lecturer for the 2007-2009 term. 